\title{
Sistema de Avaliação de TCC baseado em Lógica Fuzzy
}

\author{
Andson M. Balieiro ${ }^{3}$, Sebastião R.S. Neto ${ }^{12}$, Eraylson G. Silva ${ }^{13}$ \\ ${ }^{1}$ Universidade de Pernambuco - Campus Garanhuns (UPE) \\ Rua Cap. Pedro Rodrigues, 105 - São José - Garanhuns - PE - Brasil \\ ${ }^{2}$ Instituto de Computação - Universidade Federal de alagoas (UFAL) \\ Caixa Postal 57072-900 - Maceió - AL - Brasil \\ ${ }^{3}$ Centro de Informática - Universidade Federal de Pernambuco (UFPE) \\ Caixa Postal 50740-560 - Recife - PE - Brasil \\ \{amb4@cin.ufpe.br, srsn@ic.ufal.br, egs@cin.ufpe.br\}
}

\begin{abstract}
Resumo. O Trabalho de Conclusão de Curso (TCC) busca instigar nos alunos de graduação a pesquisa cientifica, e o desenvolvimento de habilidades importantes. A avaliação do TCC adota diversos critérios. Em geral, a soma ponderada das notas de cada critério é adotada para definir a nota final do trabalho e indicar se ele foi aprovado ou não. Este modelo de avaliação pode ocasionar a insatisfação do aluno com o resultado (ex. com nota 5.99, o TCC é considerado reprovado, enquanto que com nota 6,0 está aprovado, dado um limiar igual a 6,0). Com isso, torna-se necessário o uso de outros modelos de avaliação que realize uma transição "mais suave" entre os conceitos de aprovado. Neste aspecto, um sistema de avaliação de TCC baseado em Lógica Fuzzy é proposto. Uma avaliação de conformidade é realizada adotando casos extremos e intermediários para mostrar a sua aplicabilidade em ambientes reais.
\end{abstract}

\begin{abstract}
The end-of-graduation-course paper (EGCP) aims to motivate the students to scientific research and the development of important abilities. Its evaluation adopts several criterions. In general, the weighted sum of the values of each criterion is adopted to compute the final grade of the EGC, and indicate if it is approved or reproved. This evaluation model can lead to student dissatisfaction. Thus, it is necessary the use of alternative evaluation models that perform a "softer" transition between the approved and reproved concepts. In this respect, a system of EGCP evaluation based on Fuzzy Logic is proposed. A conformity evaluation is performed adopting the extreme and intermediate cases in order to show the applicability of the proposed system in real environments.
\end{abstract}

\section{Introdução}

O Trabalho de Conclusão de Curso (TCC), também conhecido como monografia ou trabalho de graduação, é uma forma de instigar nos alunos de graduação a realização de pesquisa científica. Através do TCC, os alunos podem desenvolver habilidades importantes como a formação de ideias, a argumentação, a busca por descobertas, empenho e foco em uma área específica, por exemplo. De acordo com [Prado 2005], o TCC "deve expressar o conhecimento do aluno sobre o assunto escolhido e é orientado por um docente da instituição". 
V Congresso Brasileiro de Informática na Educação (CBIE 2016)

Anais do XXVII Simpósio Brasileiro de Informática na Educação (SBIE 2016)

Além disso, o TCC contempla um lado prático [Kremer 1999] através do desenvolvimento de novas ferramentas e soluções. Isto exige do aluno o domínio do conhecimento abordado no decorrer da graduação e possibilita que ele coloque em prática este corpo de conhecimento na elaboração do seu trabalho. Desta forma, ele pode, além de compreender as relações entre os conteúdos vivenciados na sua graduação, verificar se está preparado para o mercado de trabalho.

Em geral, os cursos de graduações das Instituições de Ensino Superior (IES) adotam a elaboração e aprovação de um TCC como um dos requisitos para que o aluno possa concluir o curso. Este aspecto exige que tais trabalhos sejam avaliados.

A avaliação do TCC adota diversos critérios que são elaborados de acordo com as normas estabelecidas por cada IES, como pode ser observado no trabalho de [Mendes 2003]. Além disso, dentro de cada IES, os cursos podem utilizar conjuntos de critérios diferentes entre si.

Um aspecto importante na definição de critérios avaliativos é que ela norteia o aluno no desenvolvimento de um TCC com boa qualidade, que atenda aos elementos exigidos pela IES ou curso. Por outro lado, exige que os professores tenham um modelo para estabelecer pesos para cada critério e que resulte em uma nota final para o TCC. Esta nota pode indicar o nível de qualidade do trabalho, e dado um limiar de aprovação, se ele foi aprovado ou não.

Na definição de uma nota final para o TCC, em geral, os professores utilizam uma soma ponderada das notas dadas a cada critério ou uma média aritmética delas, onde um dado valor limite é definido para indicar se o trabalho foi aprovado ou não. Desta forma, uma divisão abrupta entre os conceitos é estabelecida isto pode resultar em casos onde é adotado um limiar igual a 6,0 um aluno que obtém uma nota 5,99 no TCC seja considerado reprovado, enquanto outro aluno com nota 6,00 seja considerado aprovado.

Esse modelo de avaliação ocasiona, em muitos casos, a insatisfação do aluno com o resultado. Com isso, torna-se necessário o uso de outros modelos de avaliação que realize uma transição "mais suave" entre os conceitos de aprovado e reprovado. Ademais, em processos de avaliação, é comum a adoção de termos qualificadores (valores linguísticos) como bom, ruim, excelente, por exemplo, para avaliar cada critério e posteriormente realizar operações ("média") sobre eles a fim de obter uma nota final ou conceito para uma dada atividade sendo avaliada [Senac-PE 2013].

Assim, uma alternativa para avaliar os TCCs é a utilização de sistemas inteligentes baseados em lógica fuzzy, conceito que segundo Wang [1997] tem o intuito de processar informações subjetivas de natureza vaga e incerta da linguagem natural. Um modelo de avaliação do TCC utilizando lógica fuzzy propõe um resultado com base em graus de pertinências, ou seja, um determinado aluno pode ter um trabalho classificado como ruim com grau de pertinência 0.4 e classificado como bom com um grau de pertinência 0.8 , resultando em uma nota final que seja próxima a realidade da qualidade do trabalho e transparente para o aluno.

Além disso, tais sistemas fuzzy levam em conta o conhecimento do especialista na sua operação através de suas variáveis linguísticas e conjunto de regras. Assim, o conhecimento do professor que utiliza variáveis como bom, ruim e excelente, por exemplo, e opera sobre elas para definir uma nota é possível de ser representado através de sistemas fuzzy. Desta forma, este artigo propõe um sistema de avaliação de TCC baseado em lógica fuzzy e avalia a sua conformidade através de testes com casos extremos e casos intermediários. 
V Congresso Brasileiro de Informática na Educação (CBIE 2016)

Anais do XXVII Simpósio Brasileiro de Informática na Educação (SBIE 2016)

De acordo com os aspectos acima delineados, este trabalho encontra-se assim organizado. A Seção 2 apresenta trabalhos relacionados, que adotam sistemas fuzzy na sua estrutura. Seção 3 apresenta a metodologia utilizada para definição dos critérios utilizados no sistema de avaliação proposto. O sistema fuzzy para avaliação de TCC é descrito na Seção 4. Na Seção 5 resultados de testes de conformidade do sistema são apresentados. Por fim, as considerações finais são delineadas na Seção 6.

\section{Trabalhos Relacionados}

A utilização de sistemas fuzzy no âmbito educacional ou para fins de avaliação tem recebido bastante atenção da academia. Em Rodrigues [2012] um sistema de avaliação da qualidade de serviço de uma loja online é proposto. O sistema é baseado em lógica fuzzy e analisa duas variáveis: tempo de entrega e valor do produto.

Em Toledo [2004], a avaliação de desempenho de uma Instituição de Ensino é realizada adotando uma abordagem fuzzy. Com essa abordagem, as informações qualitativas podem ser consideradas no processo de avaliação e expressas através de uma linguagem mais natural. Segundo os autores, a abordagem Fuzzy leva em consideração a maior facilidade do ser humano em lidar com termos linguísticos em vez de valores numéricos. Além disto, a lógica Fuzzy tem uma capacidade notável de tratar com verbalizações revestidas de ambiguidade.

O trabalho de Wilges [2010] implementa uma comunidade de agentes que utiliza lógica fuzzy para determinar o perfil de aprendizagem dos estudantes sob dois enfoques: prático e teórico. O objetivo dessa classificação é conduzir o estudante para uma utilização mais apropriada do ambiente de ensino-aprendizagem virtual (AVA). As variáveis de entradas do modelo proposto foram escolhidas a partir de análise estatística e o modelo foi validado através de um conjunto de dados que possui vários perfis de aprendizagem de diversos estudantes.

Uma ferramenta baseada em sistema fuzzy que auxilia no acompanhamento e avaliação do aprendizado dos alunos de cursos do sistema presencial oferecidos pela Universidade do Estado do Amazonas (UEA) é proposta em Mavezzi [2010]. Ela permite que os docentes tomem decisões metodológicas e pedagógicas necessárias visando o bom aproveitamento do aluno em uma dada disciplina. A ferramenta possibilita a obtenção de médias "fuzificadas" durante as três semanas de aula. Segundo os autores, a utilização de sistema fuzzy ocorreu devido às incertezas das informações advinhas do ambiente de aprendizagem, onde os dados coletados possuem fontes distintas. Uma se origina no valor atribuído às atividades realizadas no AVA e a outra fonte são as avaliações escritas realizadas ao final de cada semana pelos estudantes matriculados no curso.

Como apresentado, os sistemas fuzzy tem sido amplamente utilizados no âmbito educacional. Entretanto, a sua utilização não tem contemplado a avaliação de TCC.

\section{Sistema Fuzzy Proposto e Metodologia}

A estrutura do sistema fuzzy adotado na avaliação de TCC é apresentado na Figura 1. Nela se observa elementos como o fuzzificador e defuzzificador. Eles realizam o mapeamento das variáveis que assumem valores reais (numéricos) em conjuntos fuzzy de entrada (valores linguísticos) e o conjunto fuzzy de saída (valor linguístico) em valores reais, respectivamente. Além disso, tal sistema apresenta uma base de regras da forma "se-então". Tais regras buscam expressar o conhecimento do especialista sobre o problema em questão, a avaliação de TCC. A máquina de inferência utiliza tais regras e os conjuntos fuzzy de entrada para definir qual o valor linguístico da variável de saída, que posteriormente será traduzido para um valor real pelo defuzzificador [Wang 1997]. 
V Congresso Brasileiro de Informática na Educação (CBIE 2016)

Anais do XXVII Simpósio Brasileiro de Informática na Educação (SBIE 2016)

Adicionalmente, na Figura 3 se nota que o sistema proposto adota domínio, produção e adequação como variáveis de entrada e nota final como variável de saída. Estas variáveis são descritas na Seção 3.1.

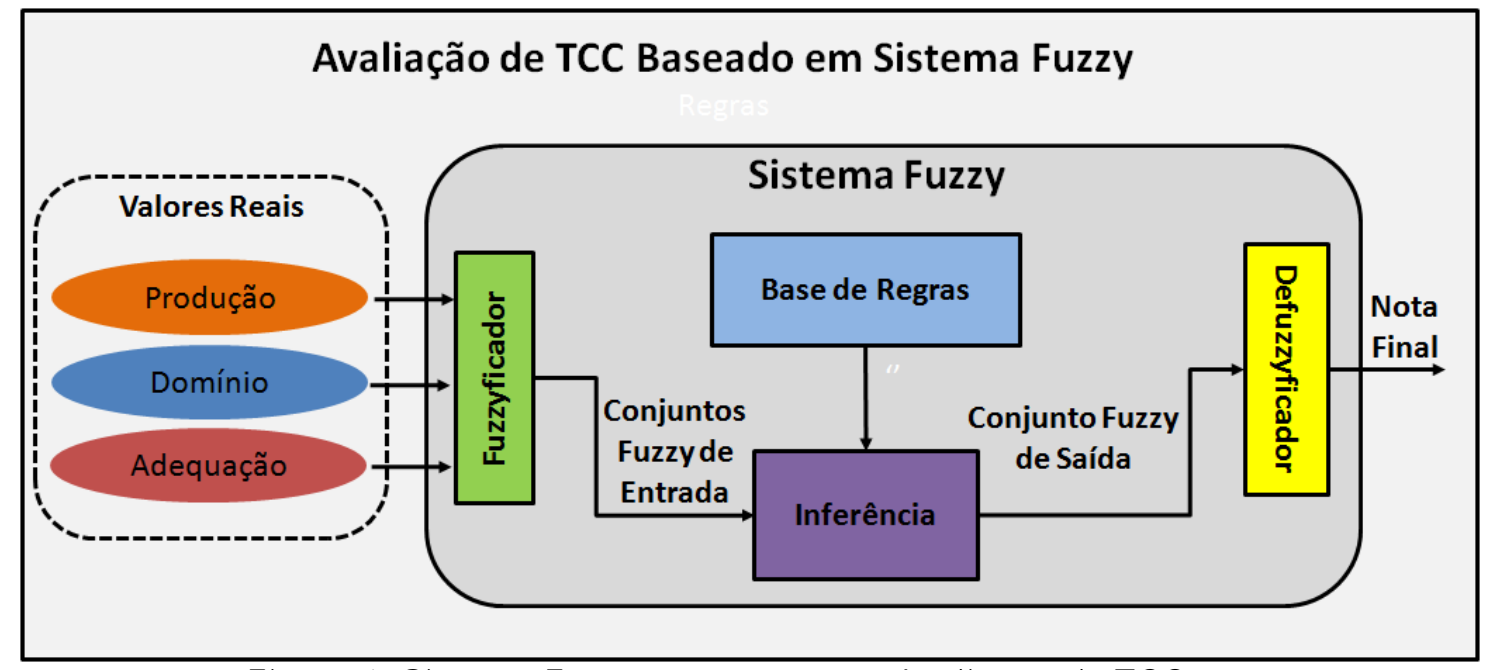

Figura 1. Sis tema Fuzzy proposto para Avaliação de TCC

\subsection{Variáveis Linguísticas}

Para definir as variáveis de entrada (critérios) a serem adotados no sistema de avaliação proposto, uma pesquisa exploratória foi realizada em sites de sete IES (UFPI, IF-Campus Valença, UEMT, IFRN, UFRA, PUC - Goiás e UESC), a fim de identificar quais os critérios são usados na avaliação de TCC dos cursos de Licenciatura em Computação e Ciência da Computação. A Tabela 1 apresenta dez critérios principais levantados.

Tabela 1. Principais critérios levantados para avaliar TCC

\begin{tabular}{|c|} 
Critérios \\
\hline Produção intelectual do aluno \\
\hline Nível de adequação do texto ao tema do trabalho \\
Clareza e objetividade do texto \\
\hline Nível de profundidade do conteúdo abordado \\
\hline Capacidade de formulação e sistematização de ideias \\
\hline Aplicação adequada da metodologia de pesquisa \\
\hline Discussão e racionalidades dos resultados apresentados \\
\hline Coerência metodológica entre a proposta e os resultados \\
\hline Habilidade de redigir e de se expressar corretamente \\
\hline Relevância das conclusões apresentadas \\
\hline
\end{tabular}

Levar em consideração todos os critérios apresentados na Tabela 1 no projeto do sistema de avaliação poderia torná-lo muito complexo. No caso do sistema fuzzy, essa complexidade se traduziria na quantidade de variáveis e conjuntos fuzzy de entrada, bem como no elevado número de regras de inferências que necessitariam ser definidas. Para contornar este problema e como alguns critérios apresentam similaridades ou objetivos próximos, eles foram divididos em três grandes categorias, as quais são: Produção, Domínio e Adequação. Desta forma, tais categorias são adotadas como variáveis linguísticas de entrada do sistema fuzzy. A Figura 2 ilustra a categorização de critérios realizada. 
V Congresso Brasileiro de Informática na Educação (CBIE 2016)

Anais do XXVII Simpósio Brasileiro de Informática na Educação (SBIE 2016)

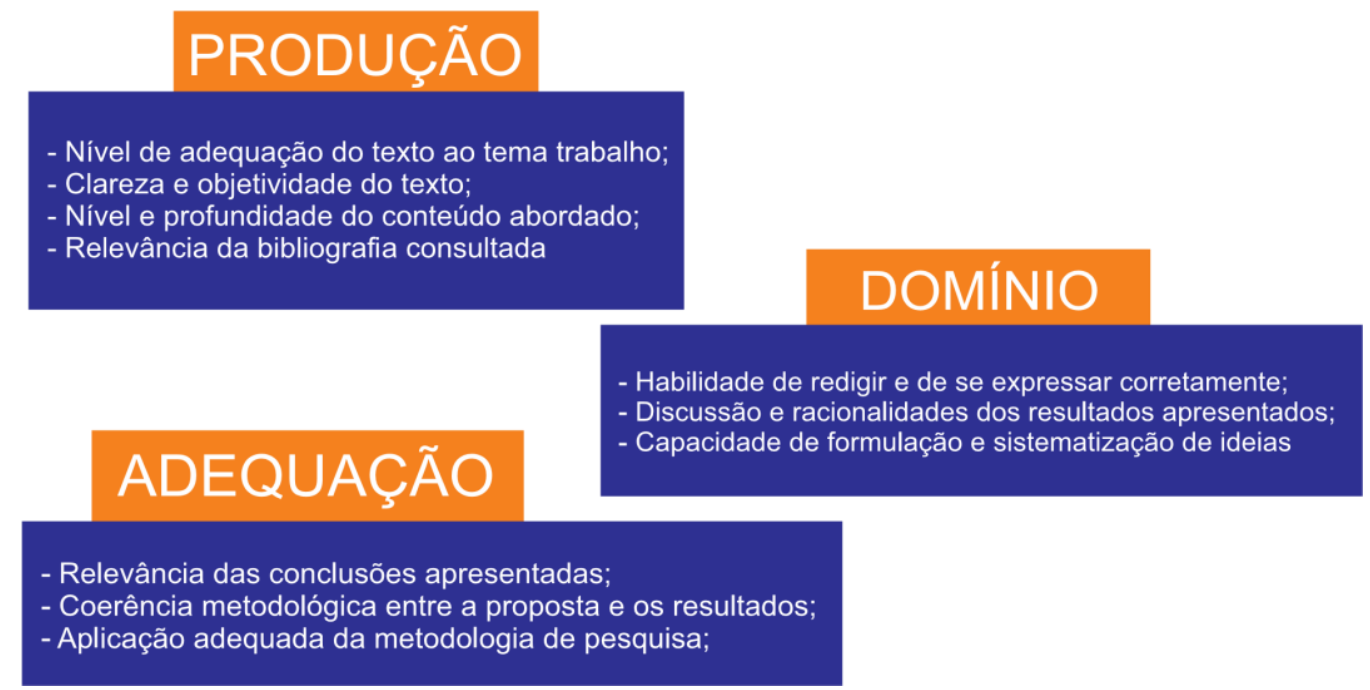

Figura 2. Divisão dos critérios em categorias

Como no final do processo de avaliação de TCC uma nota deve ser definida para expressar a sua qualidade, a variável de saída adotada no sistema fuzzy proposto foi nota final.

\subsection{Conjuntos Fuzzy das variáveis de entrada e saída}

Definidas as variáveis linguísticas de entrada e saída do sistema fuzzy, faz-se necessário definir quais os valores linguísticos elas podem assumir. Tais valores são expressos através de conjuntos fuzzy, que possuem uma função de pertinência associada. Esta função define qual o grau de penitência de um dado valor real para cada conjunto fuzzy adotado para a variável em questão [Wang 1997]. A seguir, apresentam-se os conjuntos fuzzy das variáveis de entrada e saída do sistema proposto.

Para as variáveis de entrada e saída, foram definidos quatro valores possíveis que elas podem assumir (conjuntos fuzzy), os quais são: insuficiente, regular, bom e excelente. Para representar tais conjuntos, foram adotadas funções de pertinência triangulares. O intervalo [0 10] foi definido como domínio das variáveis. As Figuras 3, 4,5,6 ilustram os conjuntos fuzzy das variáveis produção, domínio, adequação e nota final, respectivamente.

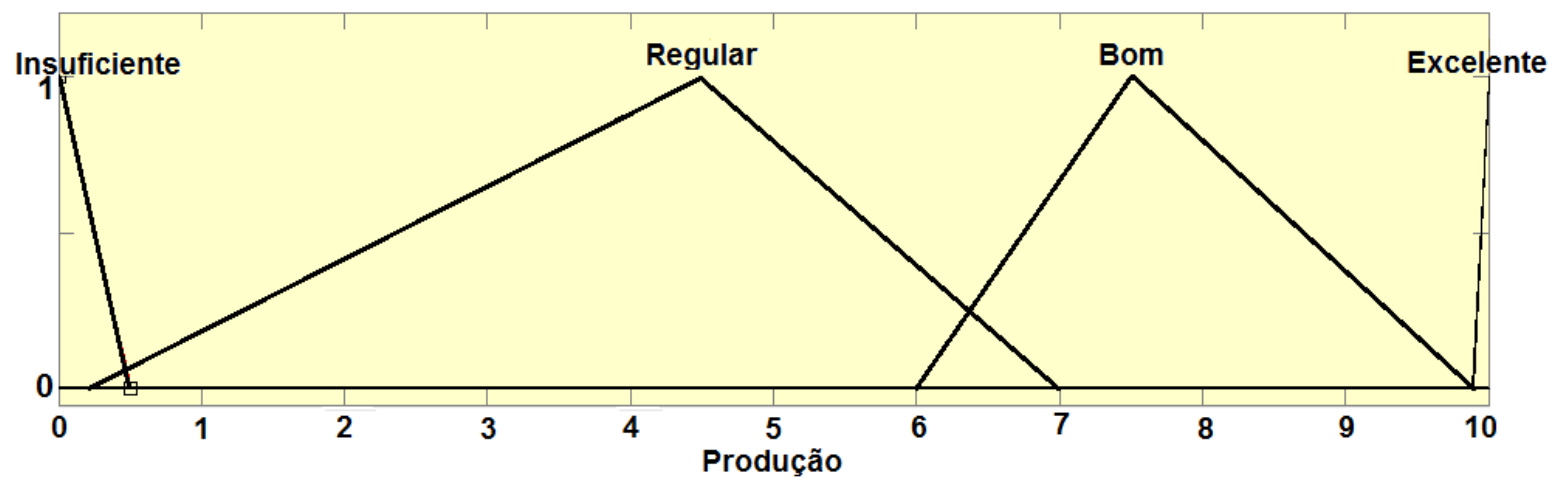

Figura 3. Conjuntos Fuzzy da variável produção 
V Congresso Brasileiro de Informática na Educação (CBIE 2016)

Anais do XXVII Simpósio Brasileiro de Informática na Educação (SBIE 2016)

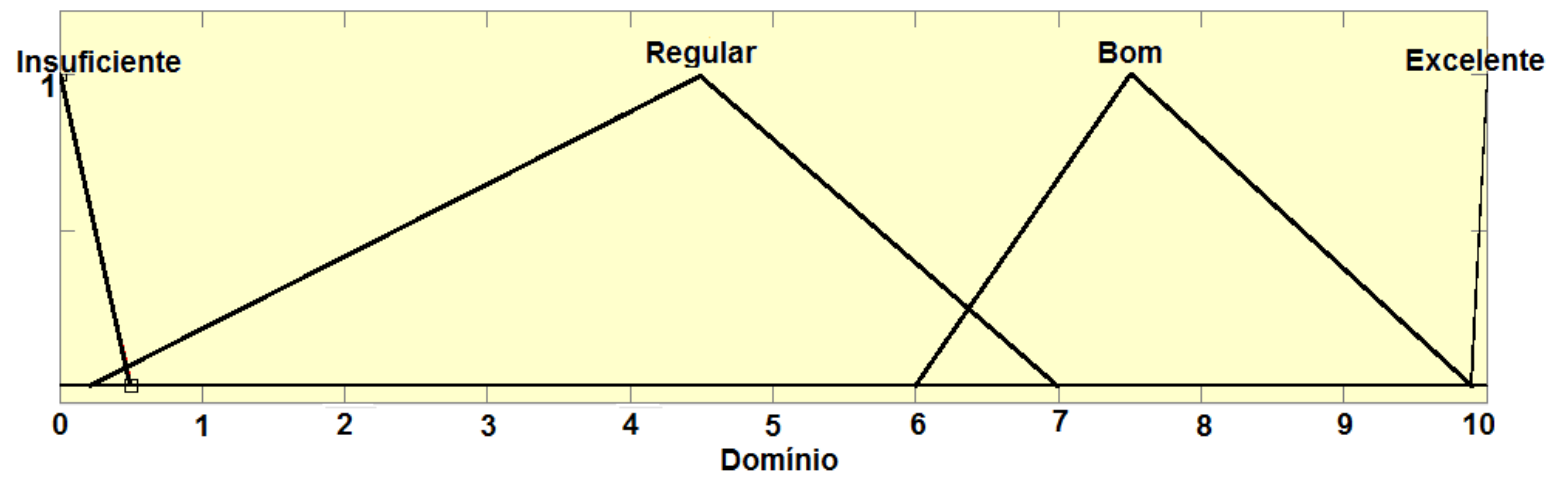

Figura 4. Conjuntos Fuzzy da variável domínio

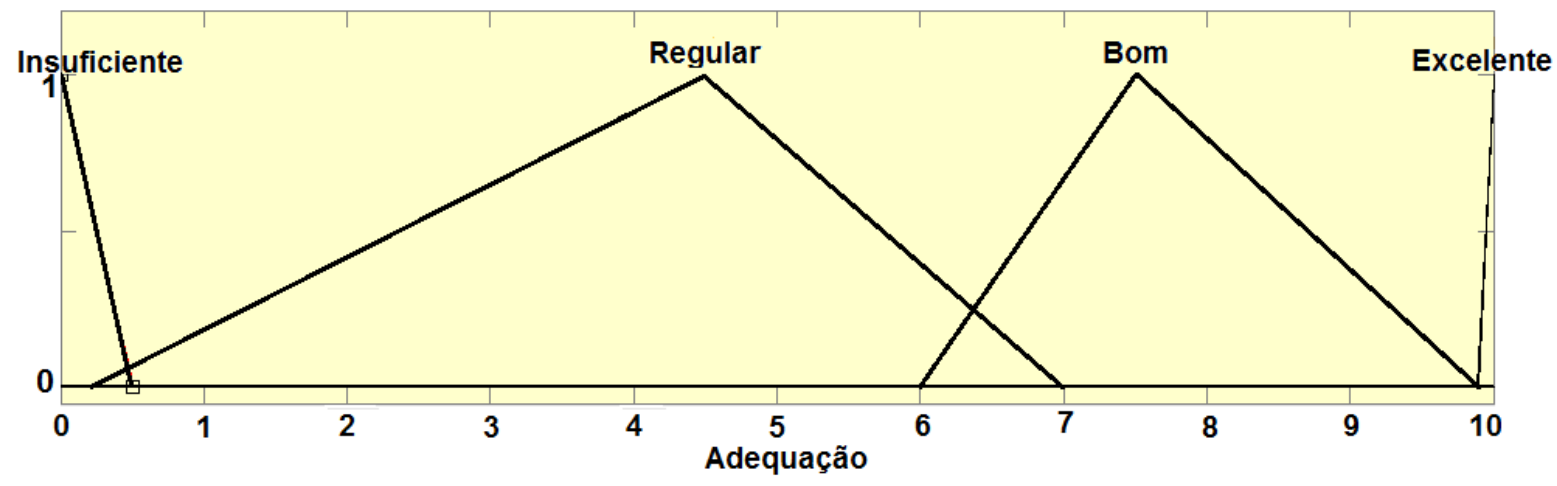

Figura 5. Conjuntos Fuzzy da variável adequação

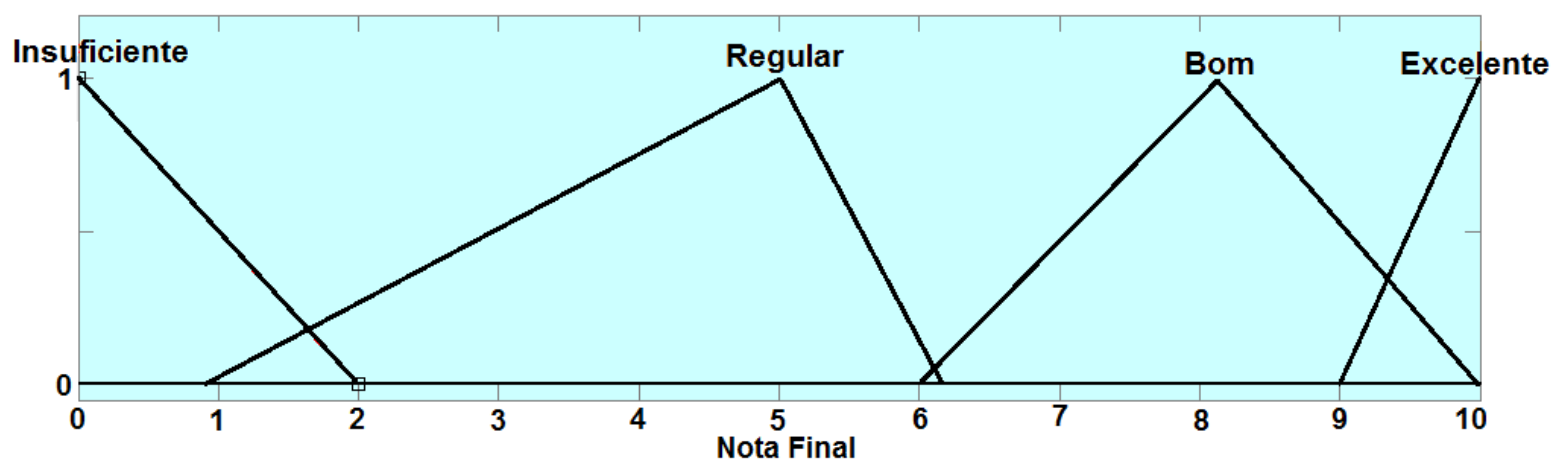

Figura 6. Conjuntos Fuzzy da variável nota final

\subsection{Fuzzificador, Defuzzificador e Máquina de Inferência}

No projeto do sistema fuzzy proposto adotou-se um fuzzificador singleton para realizar o mapeamento dos valores reais em conjuntos fuzzy. A máquina de inferência mínimo foi a selecionada. Para realizar o mapeamento de valores fuzzy para valores reais, o minddle of maximum (MOM) foi o defuzzificador adotado [Wang 1997]. Ele calcula o valor mínimo do máximo da altura do conjunto fuzzy de saída. Ele foi selecionado, pois atende a conformidade na avaliação de TCC quando se realiza testes considerando os casos de valores extremos, $(0,0,0)$ e $(10,10,10)$, por exemplo.

\subsection{Regras Fuzzy}

As regras Se-Então que compõe a base de regras do sistema fuzzy são definidas 
V Congresso Brasileiro de Informática na Educação (CBIE 2016)

Anais do XXVII Simpósio Brasileiro de Informática na Educação (SBIE 2016)

semanticamente da seguinte forma: "Se Produção é $P_{i}$ e Domínio é $D_{j}$ e Adequação é $A_{k}$ então a Nota Final $F_{l}$ ". Onde $P_{i}, D_{j}, A_{k}$ e $F_{l}$ são valores linguísticos assumidos pelas variáveis definidas na Seção 3.2. Como se têm 3 variáveis de entradas, as quais podem assumir quatro valores linguísticos distintos, logo a base de regras do sistema fuzzy é formada de 64 regras. A Tabela 2 apresenta as regras definidas no sistema fuzzy proposto.

Tabela 2. Tabela de regras do sistema fuzzy proposto

\begin{tabular}{|c|c|c|c|c|}
\hline & Produção & Domínio & Adequação & Nota Final \\
\hline 1 & insuficiente & insuficiente & insuficiente & insuficiente \\
\hline 2 & insuficiente & insuficiente & regular & insuficiente \\
\hline 3 & insuficiente & insuficiente & bom & regular \\
\hline 4 & insuficiente & insuficiente & excelente & regular \\
\hline 5 & insuficiente & regular & insuficiente & insuficiente \\
\hline 6 & insuficiente & regular & regular & insuficiente \\
\hline 7 & insuficiente & regular & bom & regular \\
\hline 8 & insuficiente & regular & excelente & regular \\
\hline 9 & insuficiente & bom & insuficiente & insuficiente \\
\hline 10 & insuficiente & bom & regular & regular \\
\hline 11 & insuficiente & bom & bom & regular \\
\hline 12 & insuficiente & bom & excelente & regular \\
\hline 13 & insuficiente & excelente & insuficiente & regular \\
\hline 14 & insuficiente & excelente & regular & bom \\
\hline 15 & insuficiente & excelente & bom & bom \\
\hline 16 & insuficiente & excelente & excelente & bom \\
\hline 17 & regular & insuficiente & insuficiente & insuficiente \\
\hline 18 & regular & insuficiente & regular & regular \\
\hline 19 & regular & insuficiente & bom & regular \\
\hline 20 & regular & insuficiente & excelente & regular \\
\hline 21 & regular & regular & insuficiente & regular \\
\hline 22 & regular & regular & regular & regular \\
\hline 23 & regular & regular & bom & regular \\
\hline 24 & regular & regular & excelente & bom \\
\hline 25 & regular & bom & insuficiente & regular \\
\hline 26 & regular & bom & regular & regular \\
\hline 27 & regular & bom & bom & bom \\
\hline 28 & regular & bom & excelente & bom \\
\hline 29 & regular & excelente & insuficiente & regular \\
\hline 30 & regular & excelente & regular & bom \\
\hline 31 & regular & excelente & bom & bom \\
\hline 32 & regular & excelente & excelente & bom \\
\hline 33 & bom & insuficiente & insuficiente & regular \\
\hline 34 & bom & insuficiente & regular & regular \\
\hline 35 & bom & insuficiente & bom & regular \\
\hline 36 & bom & insuficiente & excelente & regular \\
\hline 37 & bom & regular & insuficiente & regular \\
\hline 38 & bom & regular & regular & regular \\
\hline 39 & bom & regular & bom & bom \\
\hline 40 & bom & regular & excelente & bom \\
\hline 41 & bom & bom & insuficiente & regular \\
\hline 42 & bom & bom & regular & regular \\
\hline 43 & bom & bom & bom & bom \\
\hline 44 & bom & bom & excelente & bom \\
\hline 45 & bom & excelente & insuficiente & bom \\
\hline 46 & bom & excelente & regular & bom \\
\hline 47 & bom & excelente & bom & bom \\
\hline 48 & bom & excelente & excelente & bom \\
\hline 49 & excelente & insuficiente & insuficiente & regular \\
\hline 50 & excelente & insuficiente & regular & regular \\
\hline 51 & excelente & insuficiente & bom & bom \\
\hline 52 & excelente & insuficiente & excelente & bom \\
\hline
\end{tabular}


V Congresso Brasileiro de Informática na Educação (CBIE 2016)

Anais do XXVII Simpósio Brasileiro de Informática na Educação (SBIE 2016)

\begin{tabular}{|c|l|l|c|c|}
\hline 53 & excelente & regular & insuficiente & regular \\
\hline 54 & excelente & regular & regular & regular \\
\hline 55 & excelente & regular & bom & bom \\
\hline 56 & excelente & regular & excelente & bom \\
\hline 57 & excelente & bom & insuficiente & regular \\
\hline 58 & excelente & bom & regular & bom \\
\hline 59 & excelente & bom & bom & bom \\
\hline 60 & excelente & bom & excelente & bom \\
\hline 61 & excelente & excelente & insuficiente & regular \\
\hline 62 & excelente & excelente & regular & bom \\
\hline 63 & excelente & excelente & bom & bom \\
\hline 64 & excelente & excelente & excelente & excelente \\
\hline
\end{tabular}

\section{Teste do Sistema proposto e avaliação de conformidade}

O sistema fuzzy descrito na seção anterior foi implementado através do software Matlab [Matlab 2015]. Para avaliar o sistema fuzzy proposto, foram realizados 12 testes. Destes, para verificar a conformidade do sistema quando se analisa os casos extremos (aluno recebe nota máxima ou mínima em todos os critérios), dois testes foram executados. Os outros 10 testes consideraram casos intermediários. A Seção 4.1 apresenta os resultados dos casos extremos e a Seção 4.2 aqueles obtidos nos casos intermediários.

\subsection{Casos Extremos}

Como destacado na seção anterior, os casos extremos são aqueles onde um dado TCC recebe a nota máxima/mínima possível em todos os critérios avaliados. Nestes casos, a nota final esperada para este trabalho é a máxima/mínima possível, ou seja, 10 e 0 , respectivamente, quando se considera uma faixa de 0 a 10 para o valor da nota final.

Para o caso extremo inferior $(0,0,0)$, nota-se que para esta entrada as variáveis produção, domínio e adequação assumem o valor lingüístico (pertencem ao conjunto) ineficiente com grau de pertinência igual a 1, conforme pode ser observado na Seção 3.2. Desta forma, a regra 1 é totalmente ativada e indica que o valor linguístico da variável de saída será insuficiente com grau de pertinência 1, ou seja, todo conjunto insuficiente na variável de saída é preenchido, como ilustrado na Figura 7, e irá compor a resposta do sistema. Como apenas esta regra é ativada para esta entrada, apenas a sua contribuição é levada em conta. Após a defuzzificação com operador mindle of maximo o valor real da nota final selecionada para representar a saída do sistema é 0 . De forma similar acontece com o caso extremo superior, $(10,10,10)$, onde a regra 64 é totalmente ativada, uma vez que o valor 10 pertence ao conjunto excelente com grau de pertinência maior que zero (igual a 1 neste caso) para todas as variáveis de entrada.

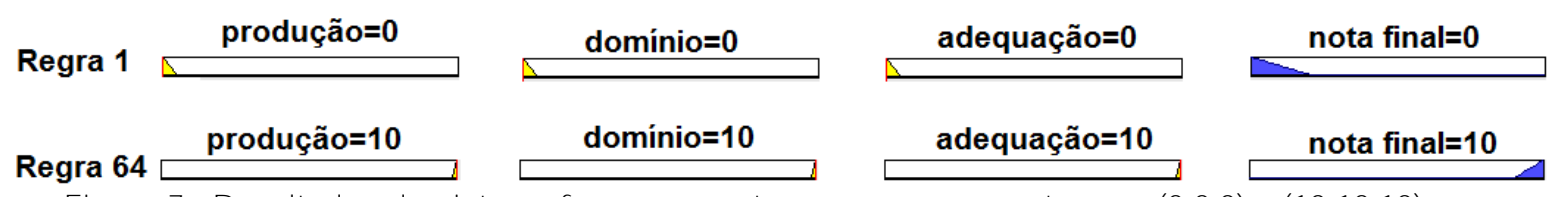

Figura 7. Resultados do sistema fuzzy proposto para os casos extremos $(0,0,0)$ e $(10,10,10)$

\subsection{Casos Intermediários}

Para definir os casos de teste intermediários, os valores reais para as variáveis de entrada (produção, domínio e adequação) foram gerados seguindo uma distribuição uniforme no intervalo de 0 a 10, que é o domínio de definição das variáveis. Desta forma, 10 casos de 
teste intermediários foram gerados, os quais estão dispostos na Tabela $3 \mathrm{com}$ os respectivos valores de saída da variável nota final dado pelo sistema fuzzy proposto.

Dentre esses casos intermediários, notam-se três casos que tiveram resultados que parecem não fazem sentido, diferentes do que se espera. O primeiro é o $(2,2,2)$ cujo valor obtido na nota final é 4,15 , onde poderia se esperar um resultado mais próximo de 2,0 . $\mathrm{O}$ segundo com entrada $(5,7,8)$ e saída 8,9 , onde uma saída próxima a 7,0 parece fazer mais sentido. Por fim, o terceiro caso com entrada $(1,9,9)$, que resulta em nota final igual 8,0, onde um resultado próximo a 6,0 poderia ser o mais adequado. Tais resultados parecem não ser adequados quando se pensa que todos os critérios têm a mesma relevância e buscase calcular a nota final através de uma média aritmética. Entretanto, como em situações reais, o sistema fuzzy implementado admite que os critérios adotados possuam níveis de importância heterogêneos, os quais foram expressos através das regras definidas. Desta forma, nota-se, por exemplo, que as regras 24 e 54 apresentam como conclusão (parte Então) valores linguísticos diferentes (bom e regular, respectivamente) para a variável nota final, apesar de elas terem no seu antecedente (parte Se) os valores linguísticos excelente, regular e regular. Esta diferença ocorre, pois, a ordem em que os valores lingüísticos são associados às variáveis é diferente, o que ilustra a heterogeneidade dos níveis de importância das variáveis de entrada na definição da nota final.

Além disso, os casos de testes intermediários buscaram mostrar o quão genérico é sistema fuzzy proposto. Como diferentes instituições podem adotar diferentes níveis de importância para os critérios de avaliação, tais diferenças podem ser facilmente incutidas no sistema fuzzy através de leves adaptações na sua base de regras, por exemplo. Isso o torna facilmente extensível para outras situações de avaliação.

Tabela 3. Casos de teste intermediários e seus resultados

\begin{tabular}{|c|c|c|c|}
\hline Produção & Domínio & Adequação & Nota Final \\
\hline 2 & 2 & 2 & 4,15 \\
\hline 7 & 7 & 7 & 8,09 \\
\hline 8 & 8 & 8 & 8,01 \\
\hline 5 & 7 & 8 & 8,09 \\
\hline 6 & 8 & 7 & 8,05 \\
\hline 8 & 4 & 4 & 4,71 \\
\hline 2 & 9 & 9 & 8,05 \\
\hline 1 & 9 & 9 & 8,02 \\
\hline 4 & 8 & 6 & 4,12 \\
\hline 5 & 5 & 5 & 4,72 \\
\hline
\end{tabular}

\section{Considerações Finais}

O TCC é um elemento importante na formação do estudante de graduação. A sua avaliação é um processo importante que adota diversos critérios, os quais podem diferir de IES para IES, e busca indicar a qualidade do trabalho elaborado. Entretanto, em geral, durante a avaliação, uma divisão abrupta entre os conceitos aprovado e reprovado pode acontecer. Neste aspecto, este trabalho apresentou um sistema de avaliação de TCC baseado em Lógica Fuzzy. Este sistema busca expressar o conhecimento dos especialistas (professores) que utilizam termos qualitativos para definir a nota final de um TCC e proporcionar uma transição mais suave entre os conceitos aprovado e reprovado. A avaliação realizada mostrou que o sistema pode ser adotado em ambientes reais e que é 
V Congresso Brasileiro de Informática na Educação (CBIE 2016)

Anais do XXVII Simpósio Brasileiro de Informática na Educação (SBIE 2016)

facilmente extensível para diferentes ambientes onde os diferentes níveis de importância dos critérios de avaliação são distintos.

Como trabalho futuro, pretende-se validar o sistema proposto, adotando dados reais obtidos através de questionários aplicados aos professores durante a avaliação de TCCs do curso de Licenciatura em Computação da Universidade de Pernambuco, campus Garanhuns. Além disso, é vislumbrado a otimização de aspectos do sistema fuzzy como as funções de pertinências e tabela de regras a fim de que os resultados do sistema casem com os resultados desejados.

\section{Referências}

Kremer, R.(1999) "Sistema De Apoio À Decisão Para Previsões Genéricas Utilizando Técnicas De Data Mining”, Trabalho de Conclusão de Curso, Bacharelado em Ciência da Computação, Universidade Regional de Blumenal.

Malvezzi, W. R.(2010) "Uma Ferramenta Baseada em Teoria Fuzzy para o Acompanhamento de Alunos Aplicado ao Modelo de Educação Presencial Mediado por Tecnologia”, In: Simpósio Brasileiro de Informática na Educação. João Pessoa: SBC.

Matlab (2015). Disponível em < http://www.mathworks.com/products/matlab/>. Acesso em Maio/2015.

Mendes, G.(2003) “Como fazer monografia na prática”, FGV Editora.

Prado, C.(2005) "Ações e projetos de um corpo docente multiprofissional em um Curso de Graduação em Enfermagem”, Tese de Doutorado, USP, São Paulo.

Rodrigues, L. M.; Dimuro, G. P. (2010) "Utilizando Lógica Fuzzy para Avaliar a Qualidade de uma Compra Via Internet", UFRGS.

Senac - PE. (2013) "Projeto Político Pedagógico", Serviço Nacional de Aprendizagem Comercial, Departamento Regional de Pernambuco, disponível em < http://www.pe.senac.br/ascom/downloads/projeto-politico-pedagogico-senac.pdf >. Acesso em Maio/2015.

Toledo, O. M., Cosenza, C. A. N. (2004) "Metodologia de Avaliação de Desempenho Baseada em Lógica Fuzzy”, COBENGE.

Wang, L-X. (1997) “A course in Fuzzy Systems and Control”, Prentice-Hall International

Wilges, B., Mateus, G., Nassar, S. M., Bastos, R. C.(2010) “Avaliação da Aprendizagem por meio de lógica de fuzzy validado por uma Árvore de Decisão ID3", CINTEDUFRGS. 